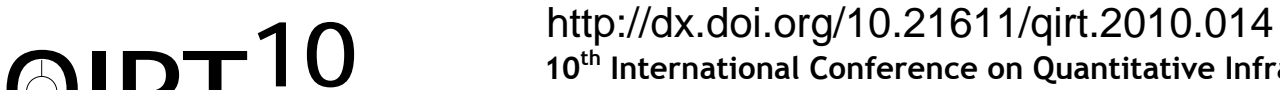 \\ $10^{\text {th }}$ International Conference on Quantitative InfraRed Thermography \\ July 27-30, 2010, Québec (Canada)
}

\section{Near infra-red imaging with the radiative transfer equation: A comparative study with finite elements}

\author{
by O. Balima*, J. Boulanger**, A. Charette* and D. Marceau* \\ *AS Dept., Université du Québec à Chicoutimi, Chicoutimi (Québec), Canada G7H 2B1, \\ Olivier_Balima@uqac.ca,Andre_Charette@uqac.ca,Daniel_Marceau@uqac.ca \\ ${ }^{* *}$ AR-GTL, National Research Council, Ottawa (Ontario), Canada K1A OR6, joan.boulanger@nrc-cnrc.gc.ca
}

\begin{abstract}
This paper presents a comparative study of near infra-red optical tomography with the radiative transfer equation. The forward model is based on the least square finite element method associated to the discrete ordinates method. Comparative tests of reconstruction on noise free data of optical properties are carried out. The results show as expected that increasing the number of degrees of freedom of the coefficients improves the quality of the reconstruction. It is also seen that using discontinuous elements for the coefficients, gives better results than continuous one as they are well suited for handling the discontinuities in the coefficients field which is usually encountered in real applications.
\end{abstract}

\section{Introduction}

In the last decade, increasing developments have been achieved in non-invasive clinical controls and medical diagnostics techniques such as magnetic resonance imaging and positron emission tomography, to name a few. Among these methods, near infrared optical tomography has been emerging as a promising diagnostic tool as this method requires a relatively low-cost system development with a reasonable accuracy compared to other methods. In addition, the method does not make any damage to biological tissues as the wavelength of the light source used for probing is a near infrared radiation in the range between 600 and $900 \mathrm{~nm}$. These developments were made possible thanks to advances in the theoretical understanding of the interaction between light and optical properties of semi-transparent media such as human tissues. On the other hand, the improvement of light detection systems as well as light sources that irradiate the tissues, was necessary in order to put these techniques into practice. For this purpose, major studies were carried out using the full radiative transfer equation [1, 2, 3, 4] as this equation properly describes light transport in biological tissues.

However, the radiative transfer equation is difficult to solve and analytical solutions are available only for simple cases. Different approximations are used, among which the diffusion approximation is well suited for biological tissues due to their high scattering [5]. While this approximation leads to an equation that is easy to solve, it fails to describe light transport near boundaries, sources, and in void-like regions where the mean free path is very large. We have developed so far a number of reconstruction algorithms for optical tomography applications with numerical models based on the radiative transfer equations in steady, frequency and time domains $[1,3,6,7,8]$. As for optimization, robust algorithms of gradient type have been developed and coupled with the adjoint method for the computation of the objective function gradient, among which we can name the conjugate gradient without line search, the sensitive function conjugate gradient [9].

Lately, we have made some developments in frequency domain optical tomography with a finite element based model of the collimated irradiation where both, the forward and the adjoint models take into account the collimated direction and where the computation of the objective function gradient with an adjoint method is introduced in a Langragian formalism that ease the understanding of the method in optical tomography applications $[10,11,12]$. This study presents a comparative study to gauge the image accuracy according to the finite element space chosen for the optical properties. We first describe the reconstruction procedure in optical tomography and then use numerical test on noise free data to compare the accuracy in the reconstruction of optical properties of a scattering and absorbing medium where different finite element spaces are used to model the optical properties. The development is done in the frequency domain. 


\section{Optical tomography}

Optical tomography is an inverse method which aims at identifying the optical properties (scattering and absorption coefficients) of a numerical model based on light transport within tissues from boundary measurements [13]. This is done by minimizing the mean square discrepancy between the measurements and the predictions of the numerical model (forward model) at detectors locations.

\subsection{Forward model equations}

In the frequency domain, the prediction of the forward model is the emerging light intensity from the medium. This prediction is given by

$$
P(r)=\int_{\vec{\Omega} \cdot \vec{n}>0} I_{s}(r, \vec{\Omega}, \omega) \vec{\Omega} \cdot \vec{n} d \Omega \quad \forall r \in \partial \mathcal{D}
$$

where the boundaries are considered as transparent and non-reflecting, $\partial \mathcal{D}$ is the boundary of the medium $\mathcal{D}$, $I_{s}(r, \vec{\Omega}, \omega)$ is the complex scattered component of the intensity at the spatial position $r, \vec{\Omega}$ is the light propagation direction, $\omega$ is the modulation frequency and $\vec{n}$ is the outward unit vector at the boundary surface. The scattered intensity $I_{s}(r, \vec{\Omega}, \omega)$ is solution of

$$
\left(\vec{\Omega} \cdot \nabla+\frac{i \omega}{c}+\kappa+\sigma\right) I_{s}(r, \vec{\Omega}, \omega)=\frac{\sigma}{4 \pi} \int_{4 \pi} I_{s}\left(r, \vec{\Omega}^{\prime}, \omega\right) \Phi\left(\vec{\Omega}^{\prime}, \vec{\Omega}\right) d \Omega^{\prime}+S_{c}(r, \vec{\Omega}, \omega)
$$

where $\kappa=\kappa(r)$ and $\sigma=\sigma(r)$ are respectively the absorption and the scattering coefficients, $\Phi\left(\vec{\Omega}^{\prime}, \vec{\Omega}\right)$ is the scattering phase function, $S_{c}(r, \vec{\Omega}, \omega)$ is the collimated source term induced by the scattering of the collimated part of the intensity within the medium. The boundary condition of Eq. (2) is

$$
I_{s}(r, \vec{\Omega}, \omega)=0, \quad \vec{\Omega} \cdot \vec{n}<0, \quad \forall r \in \partial \mathcal{D} .
$$

The collimated source term is given by [5]

$$
S_{c}(r, \vec{\Omega}, \omega)=\frac{\sigma}{4 \pi} I_{c}(r, \omega) \Phi\left(\vec{\Omega}_{c}, \vec{\Omega}\right)
$$

where $\vec{\Omega}_{c}$ is the collimated source direction, and $I_{c}(r, \omega)$ is the collimated intensity solution of the extinction law [5] such that

$$
\left(\frac{i \omega}{c}+\vec{\Omega} \cdot \nabla+\kappa+\sigma\right) I_{c}(r, \omega)=0
$$

where the boundary condition is a collimated external radiation that penetrates into the medium with direction $\vec{\Omega}_{c}$ at spatial position $r_{0}$ :

$$
I_{c}(r, \omega)=q_{0}(r, \omega) \delta\left(r-r_{0}\right), \quad \vec{\Omega}_{c} \cdot \vec{n}<0 \quad \forall r \in \partial \mathcal{D} .
$$

In order to compute the boundary measurement by Eq. (1), one has to solve numerically the forward model equations (Eq. (5) and Eq. (2)). This is done with the least square finite element formulation associated to the discrete ordinates method whose accuracy has been shown in [10].

\subsection{Forward model solution procedure}

The method used to solve the forward model equations (Eqs. (5) and (2)) is based on the discrete ordinates method associated to the least square finite element $[14,15,16]$. In this procedure, the discrete ordinates method is used to handle angular dependency whereas the finite element is used to solve the resulting spatial equations. 


\subsubsection{Discrete ordinates method}

In the Discrete Ordinates Method, integrals over solid angles are replaced by a numerical quadrature [17]. Thus, Eq. (2) is rewritten as a spatial differential equation for each discrete direction $\vec{\Omega}_{m}$ :

$$
\left(\vec{\Omega}_{m} \cdot \nabla+\frac{i \omega}{c}+\kappa+\sigma_{s}\right) I_{s}^{m}(r, \omega)=S_{c}^{m}(r, \omega)+\frac{\sigma_{s}}{4 \pi} \sum_{m^{\prime}=1}^{M} I_{s}^{m^{\prime}}(r, \omega) \Phi\left(\vec{\Omega}_{m^{\prime}}, \vec{\Omega}_{m}\right) w_{m^{\prime}}
$$

where $M$ is the number of directions of the quadrature, $w_{m}$ and $I_{s}^{m}$ are respectively the quadrature weight and the radiative intensity in direction $\vec{\Omega}_{m}$. The corresponding boundary condition writes

$$
I_{s}^{m}(r, \omega)=0 \quad \vec{\Omega}_{m} \cdot \vec{n}<0, \quad \forall r \in \partial D .
$$

Equation (7) associated to its boundary condition (Eq. (8) is an advection type equation that is to be solved for the $M$ directions of the quadrature.

\subsubsection{Least square finite element formulation}

The equations of the collimated intensity (Eq. (5)) and the scattered intensity for each direction of the quadrature (Eq. (7)) are advection type equations that can be solved by numerical methods such as the finite volume, finite difference or finite element method, among others. In the following, the least square finite element formulation is used.

Let us rewrite the equations of the collimated intensity and the scattered intensity for each direction of the quadrature as

$$
\begin{array}{ll}
\beta . \nabla u+b u=f & \forall x \in D \\
u=h & \forall x \in \partial D^{-}
\end{array}
$$

where $\beta$ is the advection direction $\left(\beta=\vec{\Omega}_{c}\right.$ or $\left.\beta=\vec{\Omega}_{m}\right), u=u(x)$ is the complex value field, $b$ is the complex extinction coefficient, $f$ the complex source term, $\partial D^{-}=\{x \in \partial D, \beta \cdot \vec{n}<0\}$ is the inflow boundary according to the advection direction $\beta$ and $h$ is the inflow boundary complex function that is applied to the system. Equation (9) is rewritten as :

$$
\begin{array}{ll}
L(u)=f & \forall x \in D \\
G(u)=h & \forall x \in \partial D^{-}
\end{array}
$$

where operators $L(u)$ and $G(u)$ are $L(u)=\beta . \nabla u+b u$ and $G(u)=u$. The least square finite element formulation of Eq. (10) writes:

Find $u \in X$ such that

$$
B(u, v)=F(v) \quad \forall v \in X
$$

where $X$ is the finite element space where the solution $u$ is searched, $B(u, v)$ and $F(v)$ are respectively the bilinear and linear forms given by

$$
\begin{aligned}
& B(u, v)=(L(u), L(v))_{D}+\epsilon(G(u), G(v))_{\partial D^{-}} \\
& F(v)=(f, L(v))_{D}+\epsilon(h, G(v))_{\partial D^{-}}
\end{aligned}
$$

where $\epsilon$ is the penalization term chosen numerically such that $\epsilon+1 \approx \epsilon$. Details on the accuracy of the solution procedure are given in [10]. 


\subsection{Forward model algorithm}

In the algorithm of the forward model, Eq. (5) is solved first for the collimated component solution and then the resulting source term (Eq. (4)) is used for the solution of the scattered part (Eq. (7)) through an iterative procedure where the initial scattered field is null. The algorithm is stopped when the maximum absolute relative error between the current and the previous iterations of the scattered intensity solution field is low, such that

$$
\max _{i, m}\left|\frac{\mathbf{I}_{\mathbf{s}, \mathbf{i}}^{\mathbf{m}, \mathbf{k}-\mathbf{1}}-\mathbf{I}_{\mathbf{s}, \mathbf{i}}^{\mathbf{m}, \mathbf{k}}}{\mathbf{I}_{\mathbf{s}, \mathbf{i}}^{\mathbf{m}, \mathbf{k}}}\right| \leq 10^{-6}, \quad \forall i, \forall m
$$

where $\mathbf{I}_{\mathbf{s}, \mathbf{i}}^{\mathbf{m}, \mathbf{k}}$ is the discretized scattered component solution in direction $m$ at iteration $k$ and $i$ is a degree of freedom of the finite element solution.

\section{Identification of the optical properties}

The optical properties are recovered through an iterative scheme which consist in minimizing an objective function. Here, a gradient type optimization is used where the objective function gradient is deduced through an adjoint formulation.

\subsection{Objective function and its gradient}

The objective function to be minimized writes

$$
J(\theta)=\frac{1}{2} \sum_{s=1}^{N_{s}} \sum_{d=1}^{N_{d}}\left\|P_{s}\left(r_{d}\right)-M_{s, d}\right\|^{2}
$$

where $\theta$ is the vector of parameters $\left(\theta=(\kappa, \sigma)\right.$ i.e the absorption and scattering coefficients), $P_{s}(r)$ and $M_{s, d}$ are the d-th detector prediction and measurement obtained with the s-th collimated source respectively, $N_{s}$ is the number of collimated sources, $N_{d}$ is the number of detectors, $\|.\|^{2}$ is a $l_{2}$ norm.

We suppose that $\kappa$ and $\sigma$ belong to the same finite element space $\theta=(\kappa, \sigma) \in \mathbf{R}^{2 N_{c}}$ where $N_{c}$ is the number of degrees of freedom of the chosen finite element space. In the following, the space of piecewise polynomial functions of degree 1 is also chosen for $\kappa$ and $\sigma$ fields. The gradient of the objective function is computed with the adjoint method with the following expressions [11] :

$$
\begin{aligned}
& \nabla J(\theta) \delta \kappa=\left(\lambda_{s} \mid I_{s} \delta \kappa\right)_{s}+\left(\lambda_{c} \mid I_{c} \delta \kappa\right)_{c} \\
& \nabla J(\theta) \delta \sigma=\left(\lambda_{s} \mid I_{s} \delta \sigma\right)_{s}+\left(\lambda_{c} \mid I_{c} \delta \sigma\right)_{c}-\left(\lambda_{s} \mid\left(\frac{1}{4 \pi} \int_{4 \pi} I\left(r, \vec{\Omega}^{\prime}, \omega\right) \Phi\left(\vec{\Omega}^{\prime}, \vec{\Omega}\right) d \Omega^{\prime}\right) \delta \sigma\right)_{s}
\end{aligned}
$$

where (.|. $)_{s}$ and $(. \mid .)_{c}$ are inner products associated to the solution space respectively of $I_{s}$ and $I_{c}$ and $\lambda_{s}=$ $\lambda_{s}(r, \vec{\Omega}, \omega), \lambda_{c}=\lambda_{c}(r, \omega)$ are the corresponding complex vectors which represent the solution of the following adjoint equation system:

$$
\begin{aligned}
& \left(-\vec{\Omega} \cdot \nabla-\frac{i \omega}{c}+\kappa_{a}+\sigma\right) \lambda_{s}-\frac{\sigma}{4 \pi} \int_{4 \pi} \lambda_{s} \Phi\left(\vec{\Omega}^{\prime}, \vec{\Omega}\right) d \Omega^{\prime}+\frac{\partial J(\theta)}{\partial I_{s}}=0 \\
& \left(-\vec{\Omega} \cdot \nabla-\frac{i \omega}{c}+\kappa_{a}+\sigma\right) \lambda_{c}-\frac{\sigma}{4 \pi} \int_{4 \pi} \lambda_{s} \Phi\left(\vec{\Omega}^{\prime}, \vec{\Omega}_{c}\right) d \Omega^{\prime}=0
\end{aligned}
$$

with null boundary conditions for both $\lambda_{s}$ and $\lambda_{c}$ as the boundary condition of the forward model does not depend on any optical parameter. We refer to references $[18,11,19]$ for more details on how to deduce the gradient of the objective function trough a lagrangian formulation. 


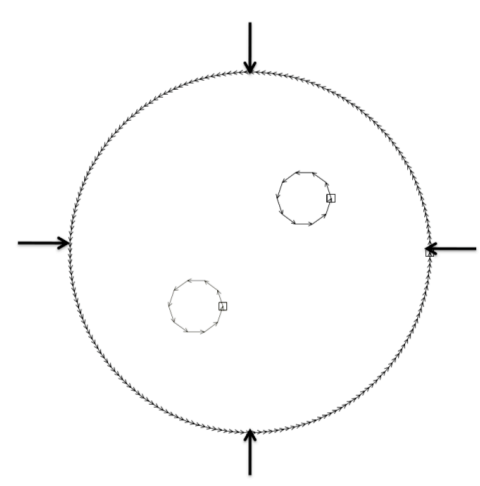

(a)

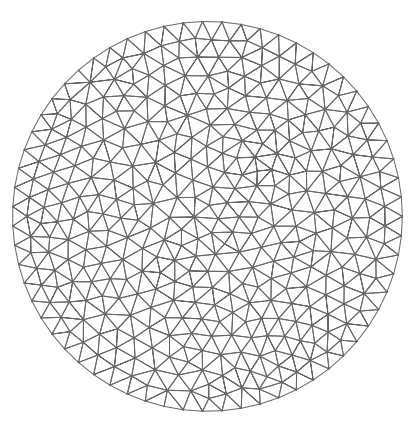

(b)

Fig. 1. Test description. (a) Two inclusions with collimated sources positions, (b) mesh of the domain.

\subsection{Inversion algorithm}

As the optical properties are generally space dependent, one may end up with a large scale minimization problem where gradient-based algorithms have shown to be efficient [9]. Here, our reconstruction scheme is based on the well known BFGS method [20,21,22] where a scaling of both the objective function and its gradient are used to handle round off erros and the low level of the boundary measurement due to the high extinction in the medium $[4,12]$.

\section{Numerical test}

\subsection{Test description}

The test medium is a circular domain of diameter of $2 \mathrm{~cm}$ which contains two inclusions (see Fig. 1(a)). Optical properties are plotted on Fig. 2 and their corresponding values are given in Table 1. The medium is assumed to be forward-scattering with a Henyey-Greenstein anisotropic factor $\mathrm{g}=0.9$, and four zero-phased illumination sources with a frequency of $600 \mathrm{~Hz}$ are regularly placed at the bottom, the right, the top and left of the medium boundary (see Fig. 1(a)). Fifty-six (56) detectors are used around the medium.

The synthetic data are generated on a regular triangular mesh of 757 elements (see Fig. 1 (b)), with $2 \times 446$ spatial degrees of freedom ( $P 1$ elements) and 48 discrete ordinates $\left(\mathrm{S}_{6}\right.$ quadrature) by using the above forward model. The generated complex-valued intensities are used as the input data for the inversion without noise for a comparative test where the coefficients $\kappa$ and $\sigma_{s}$ are approximated with different finite element spaces. In order to measure the quality of the reconstruction, we introduce an error $\epsilon$ defined by:

$$
\epsilon=\frac{1}{N_{c}} \sum_{i=1}^{N_{c}} \frac{\left(\theta_{i}^{r}-\theta_{i}^{o}\right)}{\theta_{i}^{o}}
$$

where superscripts $r$ and $o$ refer to the reconstructed and original images, respectively. The error $\epsilon$ represents the mean quadratic error per degree of freedom.

\subsection{Results}

A comparative test is carried out with different finite element spaces for the optical properties where the intensity fields are supposed to be in the space of linear continuous elements ( $P 1$ elements). Fig. 3 shows the results where the reduced scattering coefficient $\sigma_{s}^{\prime}=(1-g) \sigma_{s}$ is used. First, it is seen that the reconstruction is accurate for each case as the errors are very low (see Table 2). The errors also show that increasing the number of degrees of freedom of the coefficient improves as expected the accuracy of the image quality. A comparison of 
Table 1. Properties of the test medium

\begin{tabular}{|l|l|l|l|}
\hline & Background & Bottom inclusion & Top inclusion \\
\hline$\kappa$ & $0.5 \mathrm{~cm}^{-1}$ & $0.4 \mathrm{~cm}^{-1}$ & $0.6 \mathrm{~cm}^{-1}$ \\
\hline$\sigma_{s}$ & $50 \mathrm{~cm}^{-1}$ & $40 \mathrm{~cm}^{-1}$ & $60 \mathrm{~cm}^{-1}$ \\
\hline
\end{tabular}

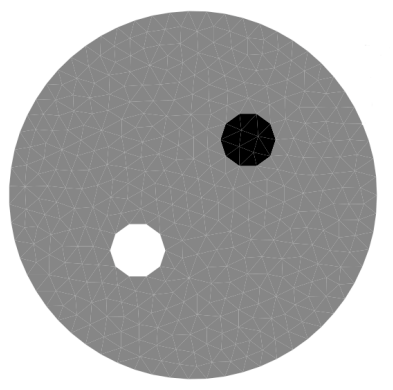

(a)

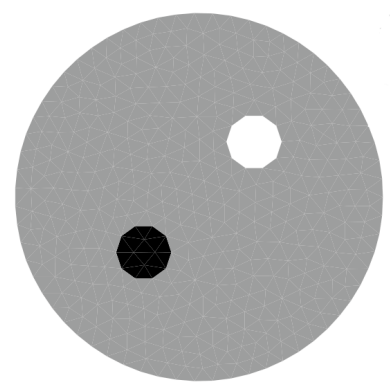

(b)

Fig. 2. Original distribution of optical properties. (a) and (b) the original distribution of the absorption and the reduced scattering coefficients respectively.

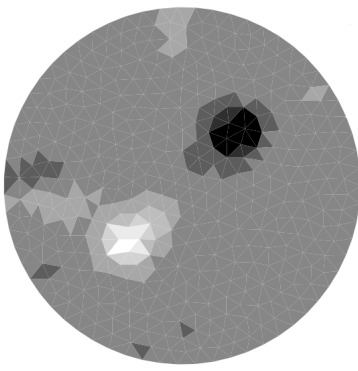

(a)

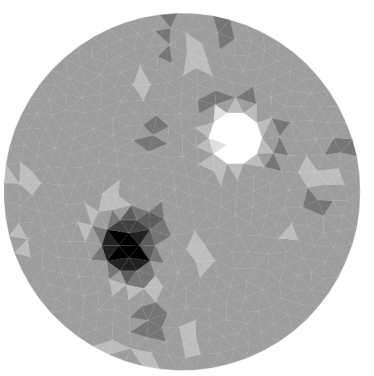

(d)

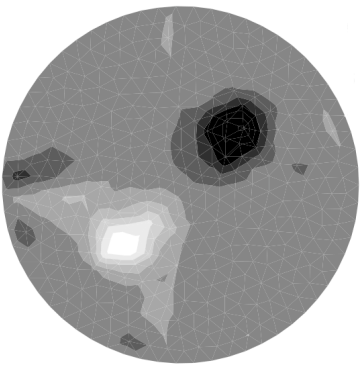

(b)

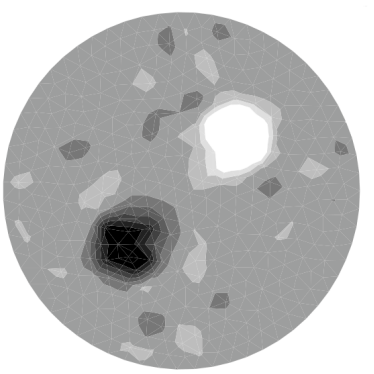

(e)

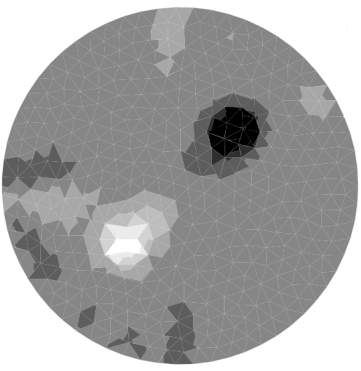

(c)

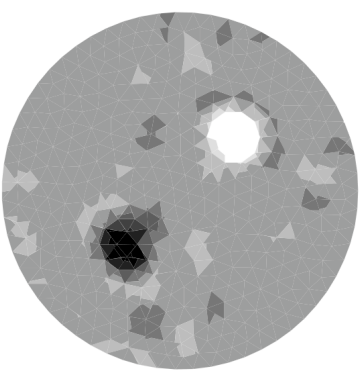

(f)

Fig. 3. Comparison of the reconstruction with linear continuous elements for the intensity fields. (a) and (d) the reconstructed image with $P 0$ elements of the absorption and the reduced scattering coefficients respectively. (b) and (e) the reconstructed image with $P 1$ elements of the absorption and the reduced scattering coefficients respectively. (c) and (f), the reconstructed image with $P 1 d c$ elements of the absorption and the reduced scattering coefficients respectively 
Table 2. Reconstruction errors where both, $\kappa$ and $\sigma$ belong respectively to $P 0$ (space of piecewise constante discontinuous finite elements) and $P 1$ (space of piecewise linear continuous finite elements). The initial objective function value is scaled with the measurement, the final value is normalized with the initial scaled objective function value. $\epsilon_{\kappa}$ and $\epsilon_{\sigma}$ are the corresponding errors for $\kappa$ and $\sigma$ coefficients respectively.

\begin{tabular}{|c|c|c|c|}
\hline Finite element space & $P 0$ & $P 1$ & $P 1 d c$ \\
\hline$N_{c}$ & 757 & 411 & 2271 \\
\hline Initial $J(\theta)$ & $1.2110^{-1}$ & $2.1510^{-1}$ & $2.2110^{-1}$ \\
\hline Normalized final $J(\theta)$ & $7.0510^{-4}$ & $1.5210^{-4}$ & $8.6810^{-4}$ \\
\hline$\epsilon_{\kappa}$ & $2.8810^{-5}$ & $6.7710^{-5}$ & $1.0110^{-5}$ \\
\hline$\epsilon_{\sigma_{s}}$ & $2.5410^{-5}$ & $5.1810^{-5}$ & $9.0810^{-6}$ \\
\hline
\end{tabular}

the image quality (Fig. 3) shows that the values of the inclusions are overestimated when the optical coefficients are assumed continuous. Therefore, discontinuous elements are better suited than continuous elements as they allow one to take into account the discontinuities of the optical properties which may arise in real application cases. Finally, we observe that the scattering coefficient is better estimated than the absorption coefficient. This is due to round off errors that affect the recovering of the absorption coefficient as its order of magnitude is very low compared to that of the scattering coefficient.

\section{Conclusion}

In this study, a comparative test of reconstruction of optical properties of scattering and absorbing medium is done. The forward model is a finite element model based on the discrete ordinates method and the least square finite method. The comparison shows as expected that increasing the number of degrees of freedom of the coefficients improve the quality of the reconstruction. It is seen that discontinuous elements give better results than continuous ones as they are well suited for discontinuous fields which is the case in real applications. Also, the low error values of the reconstruction show that the proposed method is efficient.

In a future step, we hope to speed up the forward and adjoint model by using multiprocessing solvers. A comparison will be done in order to show the influence of taking into account the collimated direction on the accuracy of the reconstruction. Also, new optimization schemes will be worked out such as the sequential quadratic programming method.

\section{REFERENCES}

[1] Kim H.K. and Charette A., "Frequency domain optical tomography using a conjugate gradient method without line search," Journal of Quantitative Spectroscopy and Radiative Transfer, 104(2), pp. 248-256, 2007.

[2] Ren K., Ball G. and Hielscher A.H., "Frequency domain optical tomography based on the equation of radiative transfer," Society for Industrial and Applied Mathematics, 28(4), pp. 1463-1489, 2006.

[3] Boulanger J. and Charette A., "Reconstruction optical tomography using transient radiative transfert equation and puls laser: a numerical study," Journal of Quantitative Spectroscopy \& Radiative Transfer, 93, pp. 325336, 2005. 
[4] Klose A., Optical tomography based on the Equation of Radiative Transfer, Ph.D. thesis, Department of Physiscs Freie Universität, Berlin Germany, 2001.

[5] Modest M.F., Radiative Heat Transfer, Mechanical Engineering, McGraw Hill, 1993.

[6] Charette A., Boulanger J. and Kim H., "Optical tomography as an inverse radiation problem," Fifth International Symposium on Radiation Transfer, Bodrum (Turkey), 2007.

[7] Boulanger J. and Charette A., "Numerical developments for short-pulsed near infra-red laser spectroscopy. part i: Direct treatment," Journal of Quantitative Spectroscopy \& Radiative Transfer, 91(2), pp. 189-209, 2005.

[8] Boulanger J. and Charette A., "Numerical developments for short-pulsed near infra-red laser spectroscopy. part ii: Inverse treatment," Journal of Quantitative Spectroscopy \& Radiative Transfer, 91(3), pp. 297-318, 2005.

[9] Charette A., Boulanger J. and Kim H.K., "An overview on recent radiation transport algorithm development for optical tomography imaging," Journal of Quantitative Spectroscopy and Radiative Transfer, 109(17-18), pp. 2743-2766, 2008.

[10] Balima O., Pierre T., Charette A. and Marceau D., "A least square finite element formulation of the collimated irradiation in frequency domain for optical tomography applications," Journal of Quantitative Spectroscopy and Radiative Transfer, 111(2), pp. 280-286, 2010.

[11] Balima O., Boulanger J., Charette A. and Marceau D., "New developments in frequency domain optical tomography. Part I. forward model and gradient computation," Sixth International Symposium on Radiation Transfer, 13-18 June, Antayla (Turkey), 2010 (accepted).

[12] Balima O., Boulanger J., Charette A. and Marceau D., "New developments in frequency domain optical tomography. Part II. application with a I-bfgs associated to an inexact line search," Sixth International Symposium on Radiation Transfer, 13-18 June, Antayla (Turkey), 2010 (accepted).

[13] Arridge S.R., "Optical tomography in medical imaging," Inverse Problems, 15(2), pp. R41-R93, 1999.

[14] Pontaza J.P., "Least-squares variational principles and the finite element method: theroy, formulations, and models for solid and fluid mechanics," Finite Elements in Analysis and Design, 41, pp. 703-728, 2005.

[15] Pontaza J. and Reddy J., "Least-square finite element formulation for one-dimensional radiative transfer," Journal of Quantitative Spectroscopy \& Radiative Transfer, 95(3), pp. 387-406, 2004.

[16] Liu L., "Least-square finite element method for radiation heat transfer in graded index medium," Journal of Quantitative Spectroscopy \& Radiative Transfer, 103, pp. 536-544, 2007.

[17] Fiveland W., "Discrete ordinates solutions of the radiation transport equation for rectangular enclosures," Journal of Heat Transfer, 106(4), pp. 699-706, 1984.

[18] Favennec Y., Girault M. and Petit D., "The adjoint method coupled with the modal identification method for nonlinear model reduction," Inverse Problems in Science and Engineering, 14(2), pp. 153-170, 2006.

[19] Balima O., Réduction de Modèle Non Linéaire par Identification : Application en thermique et comparaison avec la méthode POD-Galerkin, Ph.D. thesis, Université de Poitiers, 2006.

[20] Liu D. and Nocedal J., "On the limited memory BFGS method for large scale optimization," Mathematical Programming: Series A and B, 45(3), pp. 503-528, 1989.

[21] Nocedal J. and Wright S., Numerical Optimization, Springer, New York, 1999.

[22] Morales J., "A numerical study of limited memory bfgs methods," Applied Mathematics Letters, 15(4), pp. 481-487, 2002. 